\title{
An Observed Decline in the Amplitude of Recent Solar-Cycle Peaks
}

\author{
G.A. Chapman • G. de Toma • A.M. Cookson
}

Received: 3 March 2014 / Accepted: 10 April 2014 / Published online: 19 April 2014

(C) The Author(s) 2014. This article is published with open access at Springerlink.com

\begin{abstract}
There has been much speculation about the extended minimum between Solar Cycles 23 and 24. Cycle 24 itself has been unusually weak compared with recent cycles. We present quantitative evidence for the weakness of both Cycles 23 and, particularly, 24. The data are objective indices derived from precision photometric images obtained on a daily basis at the San Fernando Observatory. These data form the longest running, homogeneous photometric record known to us. We show sunspot areas from red images and facular/network areas from Ca II K-line images. Spot and facular area are a simple and direct measurement of the strength of solar activity. The data clearly show the decline in the amplitude of sunspot maxima for Cycles 23 and 24 compared with Cycle 22. The relative amplitudes of mean spot area for Cycles 22 through 24 are 1.0, 0.74, and 0.37, respectively. There is also an indication that the facular-to-spot area ratio has increased in Cycle 24.
\end{abstract}

Keywords Solar cycle: observations, active regions

\section{Introduction}

The last solar-cycle minimum, between Cycles 23 and 24, was one of the lowest in nearly 100 years (e.g. Bisoi et al., 2014). It was also unusually extended in time, and the decline of Cycle 23 was unusually long. Furthermore, the rise of Cycle 24 was rather slow. Because of the decrease in spot activity in Cycle 23 (de Toma et al., 2004), speculation has surrounded predictions for the peak activity of Cycle 24 . We present simple evidence suggesting a recent

G.A. Chapman · A.M. Cookson

San Fernando Observatory, Department of Physics and Astronomy, California State University, Northridge, CA, USA

G.A. Chapman

e-mail: gchapman@csun.edu

G. de Toma $(\bowtie)$

High Altitude Observatory, National Center for Atmospheric Research, Boulder, CO, USA

e-mail: detoma@ucar.edu 


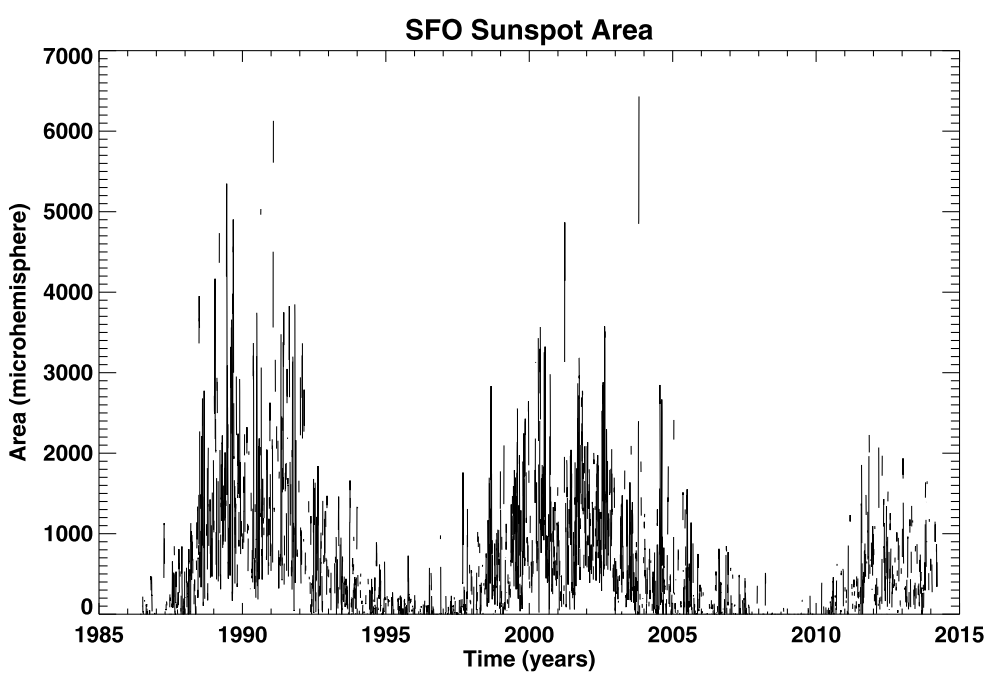

Figure 1 Daily, unsmoothed sunspot area from red CFDT1 data. The data are from mid-1986 through March 2014.

long-term decrease in solar-cycle strengths. There are a number of proxies for the strength of a solar cycle, including the International Sunspot Number (ISSN: Lefevre and Clette, 2013) and $\mathrm{F}_{10.7}$. The record of the sunspot number goes back significantly in time, but there is some uncertainty about its correct scale (Svalgaard, 2012). The 10.7-cm radio flux (Tapping and DeTracey, 1990), while an objective measurement, is a function of physical conditions in several regions of the Sun's atmosphere. For this article we have chosen, primarily, to use the area of sunspots and faculae/network determined from photometric images obtained at the San Fernando Observatory (SFO). These data are a straightforward and objective measure of surface solar activity.

\section{Description of the Data}

We present sunspot $\left[A_{\mathrm{s}}\right]$ and facular $\left[A_{\mathrm{f}}\right]$ areas for the peaks of Cycles 22, 23, and 24. Figures 1 and 2 show the daily values of the corrected sunspot and facular area. The areas are in units of $\mu$ hem (millionths of a hemisphere). These data, which have not yet been published, are available at www.csun.edu/sfo. As we show below, there is a clear, statistically significant decrease in peak amplitude with time. Figure 1 gives sunspot areas determined from Cartesian Full Disk Telescope no. 1 (CFDT1) red images taken at $672.3 \mathrm{~nm}$ with a bandwidth of $10 \mathrm{~nm}$. CFDT1 images have a pixel size of 5.12 by 5.12 arcsec. A sunspot pixel is defined as having a contrast of $\leq-8.5 \%$ on a red contrast image. A contrast image is created by removing the quiet-Sun limb darkening by fitting to the median of 20 annuli. The contrast of the $i$ th pixel is $I_{i} / I_{Q}-1$, where $I_{i}$ is the intensity of the $i$ th pixel and $I_{Q}$ is the quiet-Sun intensity at the same equivalent disk position.

Figure 2 gives the facular area from the K-line composite, described more fully below, computed using K-line data from CFDT1 and Cartesian Full Disk Telescope no. 2 (CFDT2). CFDT2 images have a pixel size of 2.5 by 2.5 arcsec. The K-line filter, $\lambda=393.4 \mathrm{~nm}$, has a bandwidth of $1 \mathrm{~nm}$. A facular pixel is defined as having a contrast of $\geq+4.8 \%$ on a K-line contrast image. 


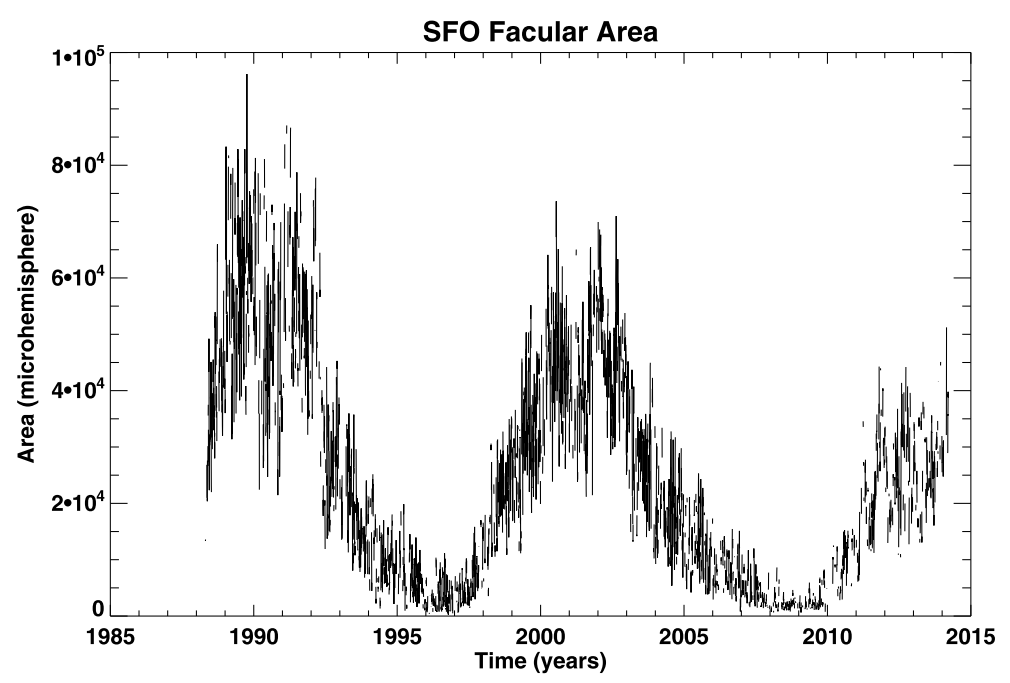

Figure 2 Daily, unsmoothed facular area from the composite K-line. The data are from mid-1988 through March 2014.

Table 1 Mean and Standard Deviation of Cycle Peak Amplitudes [ $\mu$ hem].

\begin{tabular}{llllll}
\hline Cycle & $A_{\mathrm{s}}$ & $\sigma$ & $A_{\mathrm{f}}$ & $\sigma$ & $A_{\mathrm{f}} / A_{\mathrm{s}}$ \\
\hline 22 & 1643 & 42 & 59163 & 494 & 36.0 \\
23 & 1212 & 28 & 44616 & 362 & 36.8 \\
24 & 615 & 24 & 26707 & 364 & 43.4 \\
\hline
\end{tabular}

The $A_{\mathrm{s}}$ data in Table 1 are corrected sunspot areas from CFDT1 at the San Fernando Observatory (SFO). The $A_{\mathrm{f}}$ data are corrected facular areas from the SFO K-line composite (see below). The $\sigma \mathrm{s}$ are standard deviations of the mean. The area of features is determined by removing limb darkening and searching the flattened contrast image according to the appropriate contrast criterion. The resulting areas are corrected for foreshortening on a pixelby-pixel basis. The image processing is more fully described by Walton et al. (1998).

Cycle 22 and 23 results are for three years at cycle maximum, 1989, 1990, and 1991, and 2000, 2001, and 2002, respectively. Cycle 24 results are for the last four months of 2011 (when there were some large spot groups), all of 2012, and all of 2013. Cycle 22 and 23 spot data agree well with annual sums published in Table 2 of Chapman, Dobias, and Arias (2011). Annual facular data differ somewhat because of the use of a newer K-line composite. The K-line composite is formed by least-squares fitting of overlapping segments of wide K-line data from CFDT1 and CFDT2. The newer composite is the result of an improved determination of the degradation over time in the wide K-line filters or other changes to the telescopes. The data were broken into eight intervals for a least-squares comparison of the K-line indices. For example, a light baffle was added to CFDT1 at the end of the second interval. The results of the regressions were then used to produce scaling factors to create the composite. When data existed for both telescopes, the resulting indices were averaged. Otherwise, only data from CFDT1 or CFDT2 were used. As for the new K-line composite, the older composite showed a decline in peak solar-cycle amplitude (Table 3 of Chapman, 


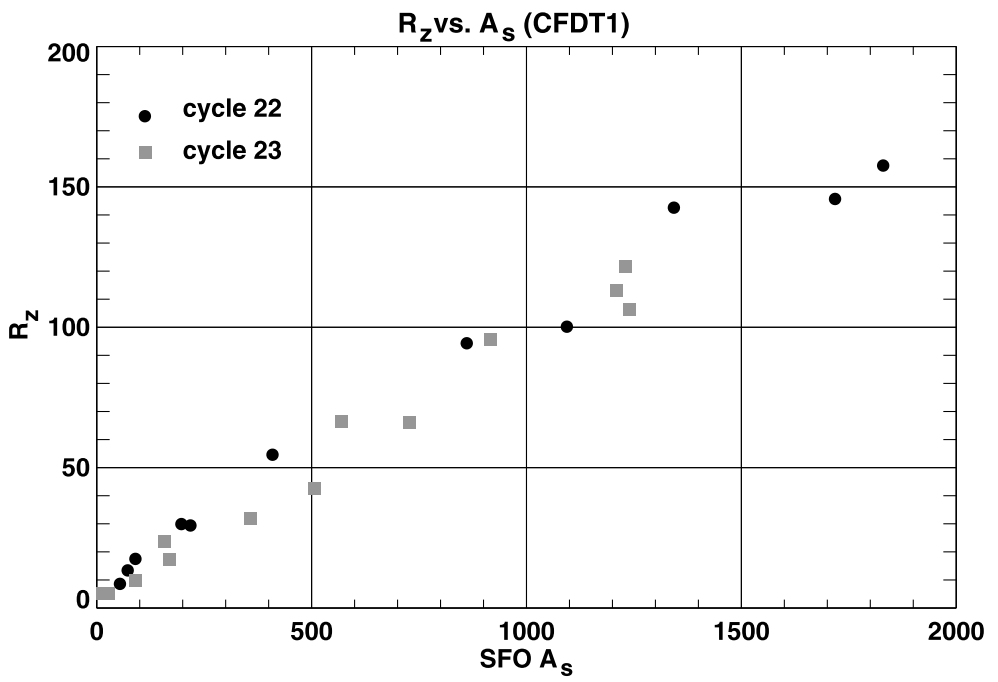

Figure 3 The annual Zurich sunspot number $\left[R_{\mathrm{Z}}\right]$ versus annual sunspot area from CFDT1. The data are from 1986 through 2009. The value of $r^{2}$ is 0.9748 (see Equation (1)). Cycle 22 data are represented by filled circles, Cycle 23 data by filled squares.

Dobias, and Arias, 2011), giving the mean facular areas of 63 872, 45200 , and 26598 for Cycles 22, 23, and 24, respectively (compare with Table 1).

Figure 3 shows $R_{\mathrm{z}}$, the annual sunspot number, versus the annual sunspot area $\left[A_{\mathrm{s}}\right]$ determined from CFDT1 images. A linear regression gives

$$
R_{\mathrm{z}}=7.2 \pm 2.5+(0.087 \pm 0.003) A_{\mathrm{s}}
$$

with the coefficient of determination $\left[r^{2}\right]=0.9748$ and the number of data points $[N]=24$. A quadratic fit gives

$$
R_{\mathrm{z}}=3.4 \pm 2.8+(0.107 \pm 0.01) A_{\mathrm{s}}-(1.2 \pm 0.6) 10^{-5} A_{\mathrm{s}}^{2}
$$

The quadratic fit gives a slightly higher $r^{2}=0.9796$ than the linear fit, but because of the added coefficient the significance of the fit was noticeably reduced.

Figure 4 shows the photometric sum $\left[\Sigma_{\mathrm{K}}\right]$, which is calculated from composite K-line data derived from CFDT1 and CFDT2 K-line images. This index is defined as

$$
\Sigma_{\mathrm{K}}=\sum_{i} \frac{\Delta I_{i}}{I} \phi(\mu),
$$

where $\Delta I_{i} / I$ is the contrast of pixel $i$, and $\phi(\mu)$ is the observed quiet-Sun limb darkening. The sum is over all pixels in the solar-contrast image. Although this index is an irradiance index, rather than an area, it includes all pixels on the disk. Thus if facular features became more scattered or fainter in Cycle 24 than in earlier cycles, this index should still measure their presence. We still see a decline in this index for Cycles 23 and 24 compared with Cycle 22. 


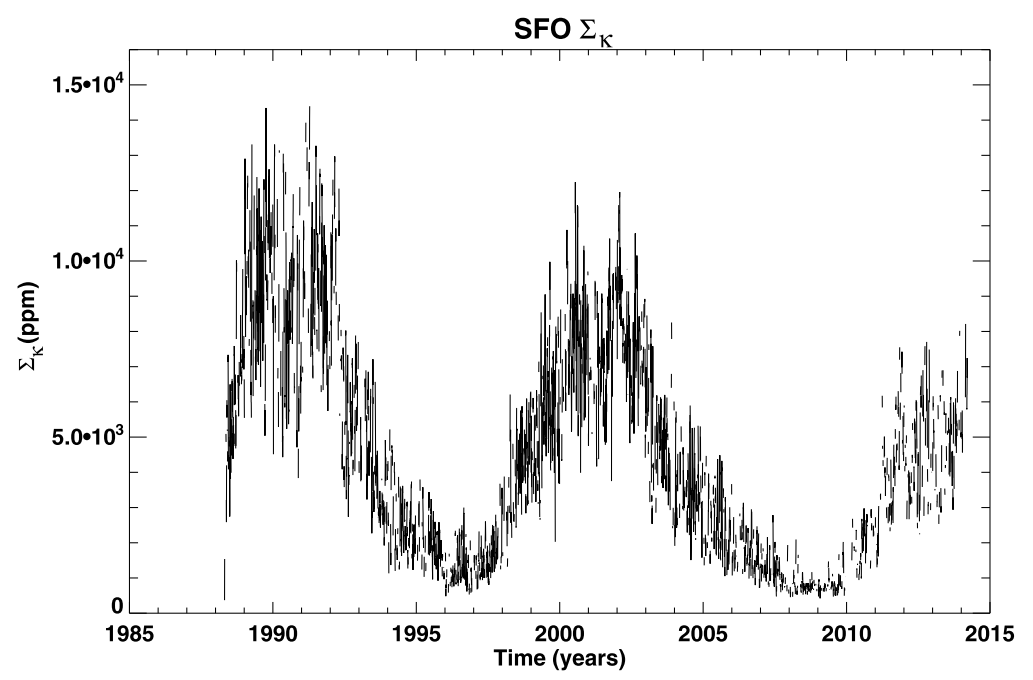

Figure 4 Temporal variation of $\Sigma_{K}$ in parts per million (ppm) of the quiet Sun. This is a K-line irradiance index, not an area. However, it includes all features no matter how faint or scattered (see Equation (3) for the definition of $\Sigma_{\mathrm{K}}$ ).

\section{Discussion}

The results of Table 1 show that the peak amplitudes of spot area and facular area are declining with time. The differences in the peaks are highly significant compared with the standard deviations of the means. The Cycle 23 spot maximum is about 10 standard deviations lower than that of Cycle 22, while the Cycle 24 maximum is about 20 standard deviations lower than that of Cycle 23. Results for Cycle 24 are, of course, preliminary as we have not seen the whole cycle. However, Cycle 24 appears to be headed for a peak amplitude of approximately one-half that of Cycle 23, in rough agreement with the predictions of Svalgaard, Cliver, and Kamide (2005), Choudhuri, Chatterjee, and Jiang (2007), Wang and Sheeley (2009), and Ahluwalia and Jackiewicz (2012). These, and many other predictions, have been nicely summarized in Hathaway (2010) and Pesnell (2012). The official NOAA prediction is that the Cycle 24 maximum would occur in May 2013, and it appears likely that Cycle 24 has reached its peak because we are already at the expected time of maximum. However, Yu et al. (2012) predicted that the peak of Cycle 24 would be January - February 2014, plus or minus six months. Moreover, the time delay between the two hemispheres in Cycle 24 suggests that Cycle 24 will have a dual peak. Cycle 24 started first, and progressed at a faster pace, in the northern hemisphere than in the southern. The mean latitude of sunspots in the two hemispheres suggests that the North has already reached its highest activity, while the South, the most active hemisphere at the time of this writing, may not yet have reached its peak. The decrease in peak cycle amplitude is also seen in the sunspot number. The average cycle maximum in sunspot number for Cycles 22, 23, and 24 is about 150, 110, and 60, respectively (www.sidc.oma.be). On the other hand, Pevtsov et al. (2014) concluded from a detailed study of sunspot magnetic fields that there has been no secular decline in the maximum magnetic field strength in sunspots, except perhaps during the last few minima.

The decline in peak activity could be part of a longer-term cycle such as the Gleissberg cycle (Gleissberg, 1967; Usoskin and Mursula, 2003; Mouradian, 2013; McCracken et al., 
2013). A multicycle modulation of 80 - 100 years opens interesting questions on the memory of solar activity. Analysis of poloidal and toroidal magnetic-field proxies over a century (Muñoz Jaramillo et al., 2013) shows that the polar flux at minimum is only correlated with the amplitude of the next cycle, indicating a short solar-cycle memory. A centennial modulation of solar activity, if real, represents a challenge for current dynamo models that cannot easily explain the presence of these long periodicities. Previously, de Toma et al. (2004) discussed the behavior of Cycle 23. The declining strength of the solar cycles shown here is not related to the projections of weakening umbral contrast and magnetic-field strength (Penn and Livingston, 2011). Their results are based on a possibly biased set of high-resolution observations of select sunspot umbrae in the infrared. They show a declining magnetic-field strength and a decreasing umbral contrast beginning in 1999. A possible explanation has been offered by Nagovitsyn, Pevtsov, and Livingston (2012). They argued that the apparent decline in field strength of sunspots is due to a changing distribution of sunspot sizes over time. A set of unbiased measurements of umbral contrast indicates that umbral contrast has not been decreasing with time, but has remained constant (Leonard and Choudhary, 2008; de Toma et al., 2013). This is consistent with the results of Pevtsov et al. (2014), who found no significant long-term trend in the maximum sunspot magnetic-field strength. Another complication is suggested by the finding that the relation between spot number and $F_{10.7}$ flux has been changing (Livingston, Penn, and Svalgaard, 2012) while the relation between $\mathrm{F}_{10.7}$ and sunspot area (de Toma et al., 2013) and $\mathrm{F}_{10.7}$ magnetic indices (Henney et al., 2012) has remained the same. On a final note, we point out that the facular-to-spot area for Cycle 24 has increased significantly, as noted in Table 1 . While this result is based on a few months near maximum, it suggests that the spot area has decreased relative to the facular area during the current cycle. We note that while the number of spots of all sizes decreased in Cycle 24 compared with the previous cycles, the decrease has been particularly severe for the large spots. This trend was already noticeable in Cycle 23, as found in our previous analysis. However, in Cycle 23 a significant reduction occurred only in the largest spots with an area larger than $700 \mu \mathrm{hem}$, while in Cycle 24 a scarcity of large spots with a more modest area in the range of $400-700 \mu \mathrm{hem}$ is also seen. This might be responsible for the observed increase in the facular-to-spot-area ratio.

Acknowledgements The observations and data analysis at SFO over the years have been carried out by CSUN students and staff. This work has been partially supported by grants from NASA (NNX11AB51G, NNX11AK46G) and NSF (ATM-0848518). The National Center for Atmospheric Research is sponsored by the National Science Foundation. We appreciate useful comments from the referee.

Open Access This article is distributed under the terms of the Creative Commons Attribution License which permits any use, distribution, and reproduction in any medium, provided the original author(s) and the source are credited.

\section{References}

Ahluwalia, H.S., Jackiewicz, J.: 2012, Adv. Space Res. 50, 662. DOI.

Bisoi, S.K., Janardhan, P., Chakarabarty, D., Ananthakrishnan, S., Divekar, A.: 2014, Solar Phys. $289,41$. DOI.

Chapman, G.A., Dobias, J.J., Arias, T.: 2011, Astrophys. J. 728, 150. DOI.

Choudhuri, A.R., Chatterjee, P., Jiang, J.: 2007, Phys. Rev. Lett. 98, 131103. DOI.

de Toma, G., White, O.R., Chapman, G.A., Walton, S.R., Preminger, D.G., Cookson, A.M.: 2004, Astrophys. J. 609, 1140. DOI.

de Toma, G., Chapman, G.A., Cookson, A.M., Preminger, D.G.: 2013, Astrophys. J. Lett. 771, 22. DOI.

Gleissberg, W.: 1967, Solar Phys. 2, 231. DOI. 
Hathaway, D.H.: 2010, Living Rev. Solar Phys. 7, 1. DOI.

Henney, C.J., Toussaint, W.A., White, S.M., Arge, C.N.: 2012, Space Weather 1002011. DOI.

Lefevre, L., Clette, F.: 2013, Geophys. Res. Abstracts 15, EGU 2013-11461-1, ADS.

Leonard, T., Choudhary, D.P.: 2008, Solar Phys. 252, 33. DOI.

Livingston, W.C., Penn, M.J., Svalgaard, L.: 2012, Astrophys. J. Lett. 757, 8. DOI.

McCracken, K.G., Beer, J., Steinhilber, F., Abreu, J.: 2013, Solar Phys. 286, 609. DOI.

Mouradian, Z.: 2013, Solar Phys. 282, 553. DOI.

Muñoz-Jaramillo, A., Dasi-Espuig, M., Balmaceda, L.A., DeLuca, E.E.: 2013, Astrophys. J. Lett. 767, 25. DOI.

Nagovitsyn, Y.A., Pevtsov, A.A., Livingston, W.C.: 2012, Astrophys. J. Lett. 758, L20. DOI.

Penn, M.J., Livingston, W.: 2011, In: Choudhary, D.P., Strassmeier, K.G. (eds.) The Physics of Sun and Star Spots, Proc. IAU Symp. 273, 126. Cambridge Journals. DOI.

Pesnell, W.D.: 2012, Solar Phys. 281, 507. DOI.

Pevtsov, A.A., Bertello, L., Tlatov, A.G., Kilcik, A., Nogovitsyn, Y.A., Cliver, E.W.: 2014, Solar Phys. 289, 593. DOI.

Svalgaard, L.: 2012, Comparative magnetic minima: characterizing quiet times in the Sun and stars. In: Mandrini, C.H., Webb, D.F. (eds.) Proc IAU Symp. 286, 27. Cambridge Journals. DOI.

Svalgaard, L., Cliver, E.W., Kamide, Y.: 2005, Geophys. Res. Lett. 32, 1104. DOI.

Tapping, K.F., DeTracey, B.: 1990, Solar Phys. 127, 321. DOI.

Usoskin, I.G., Mursula, K.: 2003, Solar Phys. 218, 319. DOI.

Walton, S.R., Chapman, G.A., Cookson, A.M., Dobias, J.J., Preminger, D.G.: 1998, Solar Phys. 179, 31. DOI.

Wang, Y.-M., Sheeley, N.R.: 2009, Astrophys. J. Lett. 694, L11. DOI.

Yu, Y., van Dyk, D.A., Kashyan, V.L., Young, C.A. 2012, Solar Phys. 281, 847. DOI. 